\title{
The Mainz Microtron MAMI —Past and future
}

\author{
A. Jankowiak ${ }^{\mathrm{a}}$ \\ Institut für Kernphysik, Universität Mainz, J.-J.-Becher Weg 45, D-55128 Mainz, Germany \\ / \\ Published online: 15 May 2006 - (C) Società Italiana di Fisica / Springer-Verlag 2006
}

\begin{abstract}
The Mainz Microtron MAMI is a cascade of three racetrack microtrons, delivering since 1991 a high-quality $855 \mathrm{MeV}, 100 \mu \mathrm{A}$ cw-electron beam for nuclear, hadron and radiation physics experiments. An energy upgrade of this facility to $1.5 \mathrm{GeV}$ by adding a Harmonic Double-Sided Microtron (HDSM) as a fourth stage is well underway and first beam is expected during the first half of 2006. A detailed description of the multiple recirculation scheme with normal conducting accelerator structures, the basis for the reliable operation of MAMI, is given and the historical development from MAMI A to MAMIB is described. The natural advancement to MAMIC by realizing a polytron of the next higher order, the HDSM, is covered in the last section and a first glimpse into the future of MAMI is given.
\end{abstract}

PACS. 29.20.-c Cyclic accelerators and storage rings - 41.75.Lx Other advanced accelerator concepts 41.85.Lc Beam focusing and bending magnets, wiggler magnets, and quadrupoles

\section{Introduction}

Since the late 1950s the electromagnetic probe had proven to be the most successful precision tool for investigating the internal structure of the atomic nucleus and hadrons. A great deal of the early experiments was done by high accelerating gradient $(\sim 20 \mathrm{MeV} / \mathrm{m})$ pulsed linacs, first with a duty factor (DF) around $0.1 \%$ and finally (Saclay, Amsterdam, MIT [1]) up to $2 \%$. However, because the capability of these latter machines for coincidence experiments was still limited, a strong demand came up in the early 1970s for continuous wave (cw, 100\% DF) high quality electron beams in the few $100 \mathrm{MeV}$ to multi GeV range ( "Lindenberger und Pinkau Ausschuß" 1980 in Germany, "Livingston Report", 1978 and "Barnes Report" 1980 in the USA ).

Two different paths to satisfy this demand were discussed - a) the pulse stretcher-ring [2], mostly designed for upgrading an existing pulsed injector, with the capability to reach energies in the some $\mathrm{GeV}$ regime, but always limited by the maximum achievable current, and b) the multiple recirculation of the beam through a linac. For this path b) again two methods were suggested: In the "independent orbit recirculation scheme" the beam is guided a few times through one or two linacs by a quite complex, but very flexible achromatic and isochronous return optics. Clearly the cw-gradient must be as high as possible and, therefore one would prefer using superconducting (sc) rf-technology. An advantage of these machines is their potential for energy upgrades - e.g., since 1975 the limit for a stable cw-operation of sc-cavities increased from ca. $4 \mathrm{MeV} / \mathrm{m}$ to $>20 \mathrm{MeV} / \mathrm{m}$ [3]. However, for a high beam

\footnotetext{
a e-mail: janko@kph.uni-mainz.de
}

quality an extraordinary amplitude and phase stability of the rf-wave is necessary.

The other possibility is to guide the beam by a few simple "combined optical elements" many times through a low gradient linac, and for this the racetrack microtron (RTM) scheme $([4,5,6])$ lends itself. Rf-gradients of 1 $\mathrm{MeV} / \mathrm{m}$ can be achieved very stable and reliable with quite low power $(\sim 15 \mathrm{~kW} / \mathrm{m})$ by conventional but highly developed normal conducting (nc) accelerating structure technology. Further, with the high beam load by many recirculations one gets a high efficiency. Because of the strong longitudinal focusing of the RTM the demands on the stability of the rf-wave are only moderate; however, realising the necessary excellent homogeneity of the magnetic field in the two $180^{\circ}$-magnets $\Delta B / B \leq 10^{-4}$ is an ambitious task.

With electron beams one has to aim for a precision of better than $1 \%$ of the very small cross sections in order to achieve meaningful physics results. Therefore, the accelerator must be expected to running reliably for up to 7000 hours a year, delivering a stable beam of excellent longitudinal and transverse emittance to the experiments. In addition, for measuring small interference effects, beams of polarised electrons are crucially important.

So in 1975 a detailed design study and the construction of an RTM-cascade MAMI was started at the Institut für Kernphysik (IKPh) at the University of Mainz [7], with the goal to realise a world class accelerator facility, capable to deliver for the first time an excellent cw-electron beam of up to about $800 \mathrm{MeV}$. At the same time first considerations on the development of a polarised photocathode gun began. 


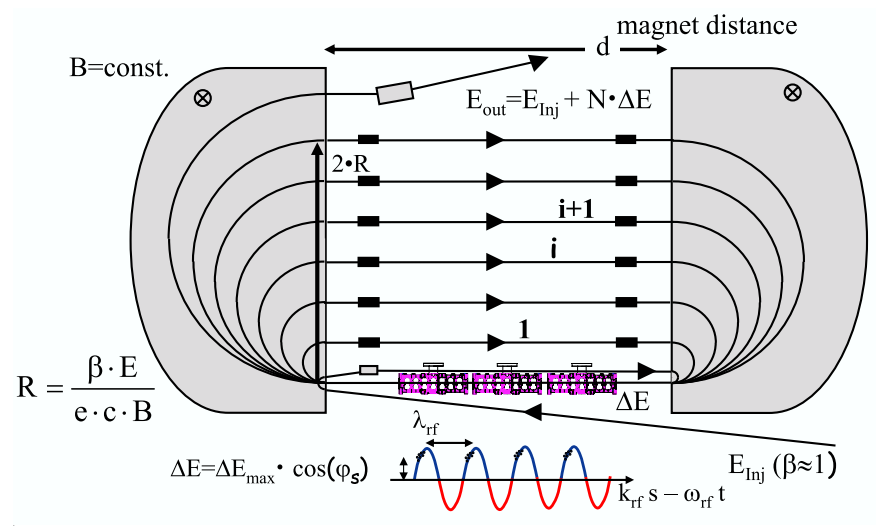

Fig. 1. Basic setup of a racetrack microtron (RTM).

\section{The racetrack microtron (RTM)}

The basic scheme of a racetrack microtron is depicted in fig. 1. For a phase coherent acceleration one has to fulfill two conditions $(\beta=v / c=1$ assumed):

a) The so called static coherence-condition for the length of the first complete circulation

$$
L_{1}=k \cdot \lambda_{r f}=\frac{2 \pi\left(E_{I n j}+\Delta E\right)}{e c B}+2 d,
$$

which must be an integer multiple of the rf-wavelength $\lambda_{r f}$ and has to be adjusted either by the injection energy $E_{I n j}$ or the distance $d$ of the $180^{\circ}$-dipoles (magnetic field $B$ ).

b) The dynamic resonance-condition for the increase in path length from turn to turn

$$
L_{i+1}-L_{i}=2 \pi \Delta R=n \cdot \lambda_{r f},
$$

which must be also an integer multiple of the rf-wavelength and is fulfilled by setting the energy gain per turn to

$$
\Delta E=\frac{e c B}{2 \pi} \cdot n \cdot \lambda_{r f}
$$

Moreover, one has to consider, that the synchronous phase range for stable longitudinal motion is given by

$$
-2<n \cdot \pi \cdot \operatorname{tg}\left(\varphi_{s}\right)<0
$$

and - for practical reasons (individual correction steering in the dispersive section) - that the distance of the return paths is $2 \Delta R=n \cdot \lambda_{r f} / \pi$.

Evidently for an ample longitudinal stability range and low rf-power consumption $n=1$ is the natural choice $\left(-32.5^{\circ}<\varphi_{s}<0\right)$. As rf-power-source a $50 \mathrm{~kW} \mathrm{cw-}$ klystron just developed for industrial heating was available in 1975 (Thomson-CSF TH2075, $\nu_{r f}=2449.5 \mathrm{MHz}$, $\left.\lambda_{r f}=12.24 \mathrm{~cm}\right)$, so that one got $2 \Delta R=3.9 \mathrm{~cm}$, enough distance for introducing slender correction steerers on each return path tube.

Concerning the beam optics the vertical defocusing in the fringe field of the $180^{\circ}$-magnets is compensated by a reversed field stripe of $-20 \% B$, so that each magnet acts like a $\pi R$ long drift. In the horizontal plane the transformation through one magnet is given by the negative unit matrix. For transverse focusing two schemes were discussed:

a) A "strong focusing" with quadrupoles on each return line, their strength adapted to the increasing beam energy to e.g. stay with constant beta-functions for each turn.

b) A "weak focusing" with only two quadrupole-doublets on the linac axis with naturally decreasing strength $\sim 1 / E^{2}$ and therefore increasing beta-functions.

It was decided to realise option b), because option a) has the disadvantage of introducing a strong transversal/longitudinal phase space coupling, and also the fabrication of very slender quadrupoles with low higher multipole content would have been difficult. Due to pseudo damping (emittance $\epsilon_{x, y}=1 /(\beta \gamma) \cdot \epsilon_{x, y}^{\text {normalised }}$ ) the beam size stays nearly constant with increasing number of turns; however the phase space ellipse gets finally quite flat. The focusing of beams of all energies simultaneously in the same quadrupole doublets is the one reason for the so called "Herminghaus rule" [7], that the ratio of output to input energy of an RTM should not exceed a factor of about 10 . The other is that at a given injection energy there is an upper limit for the magnetic field strength $B$, in order to allow for enough space for the first return of the beam backwards to pass aside the linac structures.

In order to stay within reasonable limits with the necessary strengths of the return path correctors and also to avoid noticeable distortions of the dynamic resonance condition of eq. (3), the $B$-field of the end magnets must be homogeneous to about $10^{-4}$. Because the homogeneity of the casted iron and the available mechanical manufacturing precision allows only an accuracy of some parts in $10^{-3}$, an extensive mapping of the magnetic field distribution had to be done. Based on these measurements surface correction coils were constructed, flattening the magnetic field distribution by more than a factor of ten (fig. 2, [8]).

A problem which had shown up at the first recirculators with super conducting accelerator structures ([9, 10]) was the regenerative beam blow up (BBU). If the bunched beam passes the accelerating structure slightly off axis, it excites a $T M_{110}$-like deflecting rf-mode at ca. 1.7fold the frequency of the accelerating $T M_{010}$-like mode. Its amplitude is proportional to its offset and the beam gets a small transversal kick (fig. 3). If positive feedback conditions for the next returns are given, this will lead to beam loss above a certain threshold current $I_{s}^{B B U}$. According to a "short cavity model" of this process [11] a worst case approximation is

$$
I_{S}^{B B U} \sim \frac{E_{z} \cdot \lambda_{B B U}}{(R / Q)_{B B U} \cdot Q_{B B U}} \cdot \frac{1}{\beta_{\text {foc }}} \cdot \frac{1}{N \cdot \ln \left(E_{\text {out }} / E_{\text {in }}\right)},
$$

where $(R / Q)_{B B U}$ is the shunt impedance / rf-quality factor of the BBU-mode, $E_{z}$ the accelerating field strength, $\beta_{f o c}$ the average beta-function of the recirculation, $N$ are 

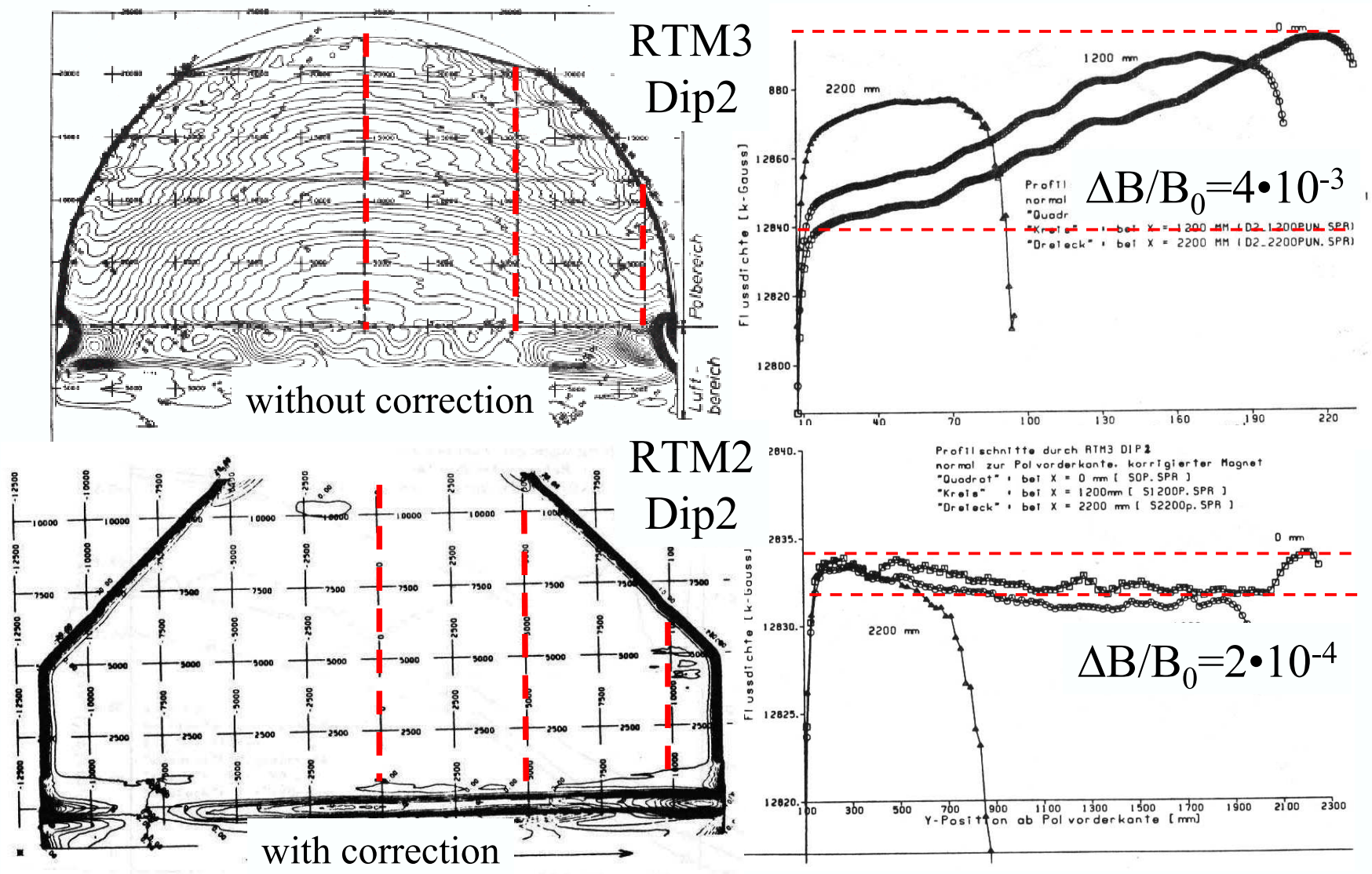

Fig. 2. Example of a field map of a RTM $180^{\circ}$-dipole magnet before and after applying the surface correction coils.

the number of turns, $E_{\text {out } / \text { in }}$ is the output/input beam energy, respectively.

Numerical BBU-calculations together with rf-measurements on the MAMI biperiodic accelerating structures [12] showed, that by staggered $T M_{110}$-detuning of the linac sections the threshold current can be shifted distinctly above the maximum design current of $100 \mu \mathrm{A}$.

\section{From MAMI A to MAMI B}

Following these design principles MAMI was realised as a 3-RTM-cascade between 1979 and 1990. Since the skepticism that a machine with many recirculations could be build a "proof of principle" was requested. Therefore, a $14 \mathrm{MeV}$ stage (MAMI A1) was built for testing and optimising the rf-structure and rf-control, the $B$-field correction by the surface coil technique and, quite advanced at that time, a complete computer control using steering algorithms. It was set into operation in March 1979 and later used as the first RTM (RTM1) of MAMI A and MAMI B. This machine was already used for first physics experiments [13] from November 1979 on. Only the klystron TH2075 and the Van-de-Graaff injector were bought, otherwise it consisted completely of in-house-made or used components as, e.g., the end magnets from DESY, Hamburg.
Originally, the second stage RTM2 was planned with an end energy of only $100 \mathrm{MeV}$ with one klystron TH2075 feeding RTM1 and RTM2 [7]. However, because of a strong demand to surpass already with this machine distinctly the pion production threshold, it was decided to add a second klystron and accelerate in RTM2 by a factor of thirteen from $14 \mathrm{MeV}$ to $180 \mathrm{MeV}$. The cost increase for the larger end magnets could be lowered by using the iron of the Heidelberg Cyclotron in use from 1943 to 1973 [14]. This setup (Van-de-Graaff + RTM1 + RTM2 $\equiv$ MAMI A) delivered from July 1983 to October 1987 about $70 \%$ of its 18700 hours of beam time for hadron and nuclear physics experiments. The maximum achieved beam parameters were $187 \mathrm{MeV}$ beam energy and a current of $65 \mu \mathrm{A}$. Its operation was funded since 1984 by a Collaborative Research Centre (SFB201, "Medium energy physics with the electromagnetic interaction") and the main components were bought via the HBFG funding.

A somewhat weak point of MAMIA was the cheap Van-de-Graaff injector. Its maximum usable voltage of only $2.1 \mathrm{MV}(\beta=0.981)$ caused a migration of the operating phase in RTM1 from $+15^{\circ}$ to $-22^{\circ}$, resulting in a reduced longitudinal acceptance and stability. This adverse effect was enhanced by the high sensitivity of the stability of the high voltage of the Van-de-Graaff to any impact of background $\gamma$ radiation. Because this background 


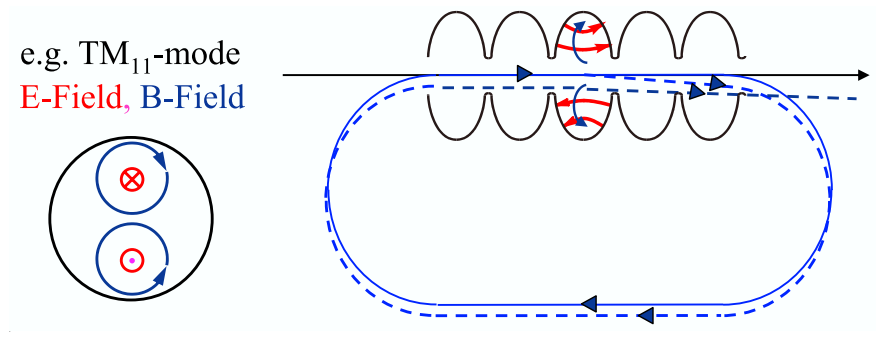

Fig. 3. Simplified sketch of the regenerative beam blow up (BBU) mechanism.

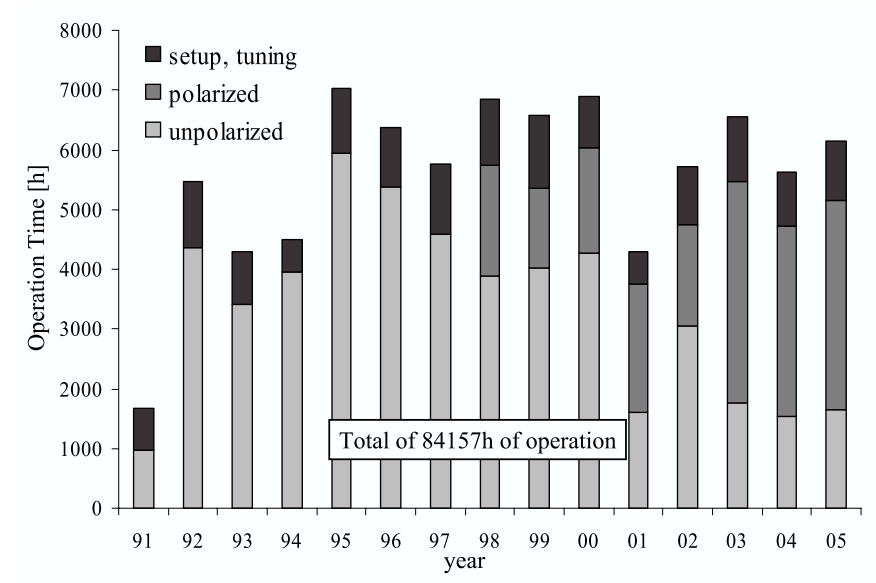

Fig. 4. Annual operation time of MAMI, according to machine setup (tuning and development), polarised and unpolarised beam time, for the years 1991 to 2005. It should be noted that in 2001 a half year shutdown took place for the preparation of the beam lines for MAMIC. Of the remaining 4428 hours the MAMI was operating for 4277 hours, i.e. $97 \%$.

radiation increased with increasing beam current, the voltage instabilities of the Van-de-Graaff were the reason for the limit of $\max .65 \mu \mathrm{A}$ beam current. Moreover, the mediocre vacuum conditions and bad accessibility of the high voltage terminal were evidently prohibitive for any operation of a GaAs-photocathode source of polarised electrons.

Therefore, when transferring MAMI A as the injector for RTM3, the Van-de-Graaff was replaced by a $3.5 \mathrm{MeV}$ linac designed and built in-house [15] with high energy stability $(\leq 1 \mathrm{keV} ; \beta=0.992)$. At this energy the phase migration in RTM1 is only $-12^{\circ}$ to $-22^{\circ}$.

The final scheme realised as MAMIB, set up in new halls from 1987 to 1990 with first operation in August 1990 , is shown in fig. 12, and its main data are given in table 1.

Apart from its excellent beam parameters (table 1), the machine showed an extraordinarily stable and reliable operation. The beam time over the years of operation since 1991, classified for machine setup and polarised and unpolarised operation for experiments, is shown in the histogram of fig. 4. The high efficiency of MAMI is due on one side to the inherent properties of the RTM, but also to a considerable extent to a sophisticated monitor sys-
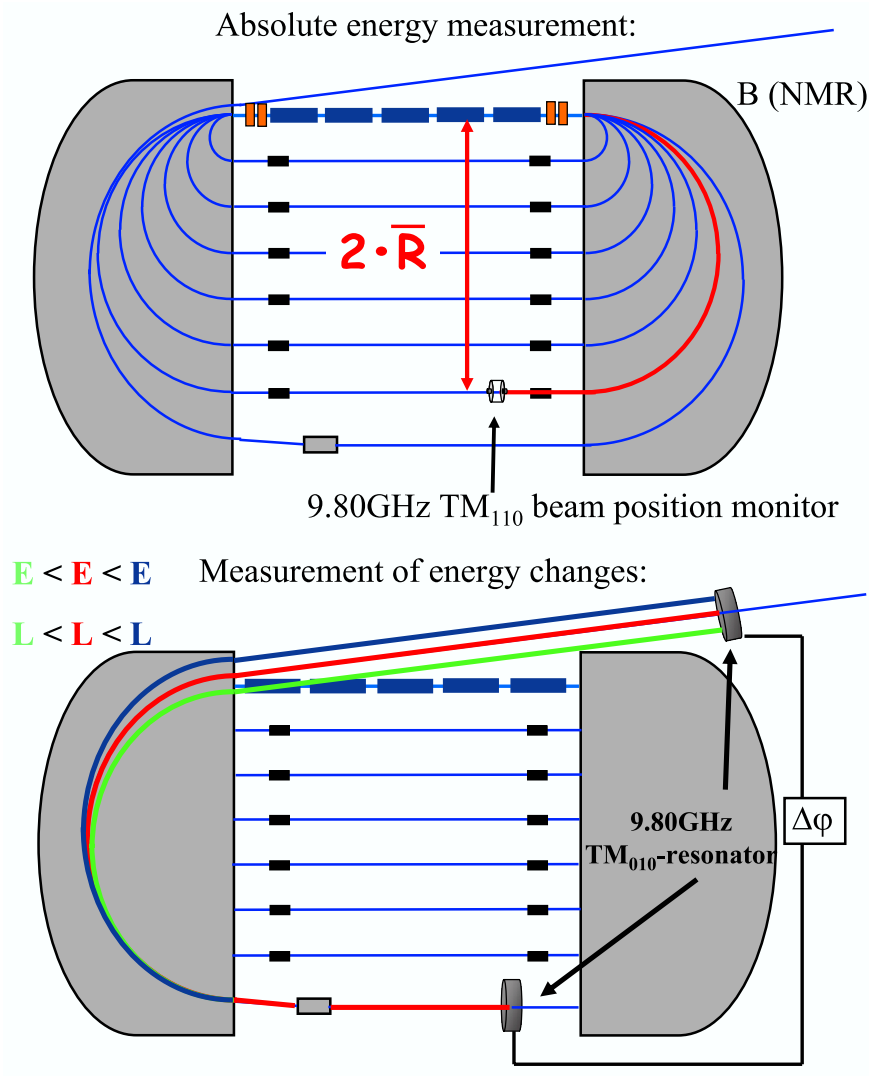

Fig. 5. Simplified scheme of the measurement of the absolute beam energy (top) and beam energy fluctuations (bottom) in RTM3.

tem [16], allowing to computer control a wide diversity of parameters and feedback loops. Among the most important monitors are the low- $Q$ rf-cavities on the linac axes of each RTM. They allow by injection of $10 \mathrm{~ns}$ diagnostic beam pulses during machine setup, actually realised as $10 \mathrm{~ns}$ blackouts in the cw-operation, to supervise the transverse positions and the phase and intensity of the beam for each recirculation individually at the entrance and exit of the linac. This information make quick and efficient correcting actions possible by a machine model implemented in the computer control system [17].

The beam profile is viewed turn by turn and in the transfer lines between the RTMs via a synchrotron radiation camera system. Very helpful for fast tuning of the matching of the beam parameters is a synchrotron radiation camera with high magnification looking through the axes of the linacs of RTM2 and RTM3. All 51 (RTM2) and 90 (RTM3) turns have to overlap and any mismatch can easily be detected and globally corrected. A system of many $T M_{110}$-rf-cavities allows a control of the beam position in diagnostic-pulse and also cw mode (above $1 \mu \mathrm{A}$ beam current) down to a few $\mu \mathrm{m}$.

The RTM configuration easily permitted in RTM3 the installation of two control setups extremely valuable for precision experiments (fig. 5). The distance between the return pipes is such that small $4 \times 2.45 \mathrm{GHz}=9.8 \mathrm{GHz}$ 
Table 1. Main parameters of MAMI. MAMIC comprises the injector linac, MAMIA, MAMIB and the HDSM being constructed.

\begin{tabular}{|c|c|c|c|c|c|c|}
\hline & & Injector & RTM1 & RTM2 & RTM3 & HDSM \\
\hline $\begin{array}{l}\text { General } \\
\text { injection / extraction energy (total) } \\
\text { number of turns } \\
\text { total power consumption }\end{array}$ & $\begin{array}{l}{[\mathrm{GeV}]} \\
{[\mathrm{kW}]} \\
\end{array}$ & $\begin{array}{c}0.511 / 3.97 \cdot 10^{-3} \\
- \\
92 \\
\end{array}$ & $\begin{array}{c}3.97 / 14.86 \cdot 10^{-3} \\
18 \\
92 \\
\end{array}$ & $\begin{array}{c}14.86 / 180 \cdot 10^{-3} \\
51 \\
220 \\
\end{array}$ & $\begin{array}{c}0.180 / 0.855 \\
90 \\
650 \\
\end{array}$ & $\begin{array}{c}0.855 / 1.5 \\
43 \\
1400\end{array}$ \\
\hline $\begin{array}{l}\text { Rf-System } \\
\text { energy resp. energy gain / turn } \\
\text { frequency } \\
\text { linac length (electrically) } \\
\text { number of sections / klystrons } \\
\text { power dissipation / beam power } \\
\text { power consumption }\end{array}$ & $\begin{array}{c}{[\mathrm{MeV}]} \\
{[\mathrm{GHz}]} \\
{[\mathrm{m}]} \\
\\
{[\mathrm{kW}]} \\
{[\mathrm{kW}]} \\
\end{array}$ & $\begin{array}{c}3.5 \\
2.4495 \\
4.93 \\
3 / 1 \\
33.2 / 0.35 \\
90 \\
\end{array}$ & $\begin{array}{c}0.599 \\
2.4495 \\
0.80 \\
1 / 1 \\
7.9 / 1.1 \\
90 \\
\end{array}$ & $\begin{array}{c}3.24 \\
2.4495 \\
3.55 \\
2 / 2 \\
48.4 / 16.6 \\
180\end{array}$ & $\begin{array}{c}7.50 \\
2.4495 \\
8.87 \\
5 / 5 \\
102.5 / 67.5 \\
450\end{array}$ & $\begin{array}{c}16.58-13.66 \\
4.8990 \mid 2.4495 \\
8.57 \mid 10.1 \\
8 / 4 \mid 5 / 5 \\
299 / 65 \\
1000^{a} \\
\end{array}$ \\
\hline $\begin{array}{l}\text { Magnet-System } \\
\text { flux density (within the gap) } \\
\text { gap height } \\
\text { min./max. deflection radius } \\
\text { iron / copper weigth of the magnets } \\
\text { number of corrector magnets } \\
\text { number of quadrupoles and solenoids } \\
\text { power consumption }\end{array}$ & $\begin{array}{c}{[\mathrm{T}]} \\
{[\mathrm{cm}]} \\
{[\mathrm{m}]} \\
{[\mathrm{t}]} \\
\\
{[\mathrm{kW}]}\end{array}$ & $\begin{array}{l}- \\
- \\
- \\
- \\
40 \\
20 \\
2 \\
\end{array}$ & $\begin{array}{c}0.1026 \\
6 \\
0.129-0.482 \\
4 / 0.2 \\
72 \\
2 \\
2 \\
\end{array}$ & $\begin{array}{c}0.5550 \\
7 \\
0.089-1.083 \\
90 / 2.3 \\
204 \\
4 \\
40 \\
\end{array}$ & $\begin{array}{c}1.2842 \\
10 \\
0.467-2.216 \\
900 / 11.6 \\
360 \\
4 \\
200 \\
\end{array}$ & $\begin{array}{c}1.53-0.95 \\
8.5-13.9 \\
2.23-4.60 \\
1000 / 27.4 \\
2 \cdot 172+2 \cdot 6 \\
2 \cdot 4 \\
400 \\
\end{array}$ \\
\hline
\end{tabular}

${ }^{a}$ Including the power consumption of one matching section between RTM3 and HDSM

${ }^{b}$ Simulation with SYTRACE, a particle tracking program including effects of stochastic emission of synchrotron radiation photons.

- $T M_{010^{-}}$and $T M_{110^{-}}$-resonators can be inserted there $\left(T M_{110^{-}}\right.$-cavities at 720 and $855 \mathrm{MeV}$ and $T M_{010^{-}}$-cavities at $315,420,510,570$ and $855 \mathrm{MeV})$. So firstly, with the $T M_{110}$-position monitor and the known distance between this monitor and the linac axis, one can use one $180^{\circ}$ dipole with its NMR-controlled field strength and precisely measured field map as a sensitive spectrometer with large $2.2 \mathrm{~m}$ bending radius to determine the absolute beam energy to $\pm 2 \cdot 10^{-4}( \pm 140 \mathrm{keV}$ at $855 \mathrm{MeV})$. Secondly, with an additional $9.8 \mathrm{GHz} T M_{010}$-cavity on the extraction path and monitoring its phase difference to one of the return-pipe $T M_{010}$-resonators, one can precisely measure changes of the length of the last half turn after extraction of the respective energy. The corresponding energy change of the electron bunches is given by eq. (2) and the sensitivity reads

$$
\frac{\lambda_{r f} / 2}{\Delta E / \text { turn }}=\frac{61.2 \mathrm{~mm}}{7.5 \mathrm{MeV}}=8.16 \mathrm{~mm} / \mathrm{MeV},
$$

which corresponds to $96^{\circ}$ phase per $\mathrm{MeV}$ at $9.8 \mathrm{GHz}$. With a resolution of $0.1^{\circ}$ at $9.8 \mathrm{GHz}$ energy changes of about $1 \mathrm{keV}$, corresponding to $1.2 \cdot 10^{-6}$ at $855 \mathrm{MeV}$, are detected. A further increase of resolution seems not to be reasonable at the moment since fluctuations of the beam direction are producing signal levels of about the same amount. By feeding back the energy signal to the linac phase, it is possible to routinely provide this energy stability of $\pm 1 \mathrm{keV}$ during physics experiments. Of course, correct tuning and a sufficient stability of the longitudinal $Q$-value are key to the well functioning of the system [18]. This setup was of fundamental importance for the parityviolating electron scattering experiments, where the cross section change with $E_{\text {beam }}^{-5}$ and must be measured with a relative precision of $10^{-6}$.
The development of the polarised source [19] after MAMI ran with a clean linac as injector is listed in the following time table:

1992 - 1995: First experiments for the A3-collaboration, beam current $I=5 \mu \mathrm{A}$, polarisation $P=30-$ $40 \%$. The $100 \mathrm{keV}$ source was installed at ground level meaning a $14 \mathrm{~m}$ long beam line to the injector linac [20].

1995: Introduction of strained layer cathodes, $I=$ $2 \mu \mathrm{A}, P=75 \%$.

1997: The source moves to the accelerator hall, which allows a much easier and reproducible injection of the beam into the linac. Installation of a $2 \mathrm{f}$-prebuncher with $4.9 \mathrm{GHz}$ [21], which allows for $145^{\circ}$ longitudinal phase space acceptance instead of the design value of $40^{\circ}[15], I=10 \mu \mathrm{A}, P>75 \%$.

1998: Introduction of the so called synchro-laser for "pulsing" the source, which allows for 90\% transmission of the precious polarised electrons, $I=20 \mu \mathrm{A}$, $P=80 \%$.

2001: Introduction of the mask activation technique, which strongly reduces losses of electrons starting at the cathode due to stray light and thus improves the vacuum conditions at the cathode. The charge extracted in one run was increased from $22 \mathrm{C}$ to $115 \mathrm{C}$. From then on several weeks of continuous operation at high current $I>20 \mu \mathrm{A}$ were possible.

2003 - 2005: A Wien filter as spin-rotator at $100 \mathrm{keV}$ electron energy directly behind the polarised source was installed. This allows for a much easier adjustment of the beam polarisation at the target for all experimental stations and all energies. Before, the spin direction at the experiments was controlled by tuning the MAMI end energy making use of the gyromagnetic 


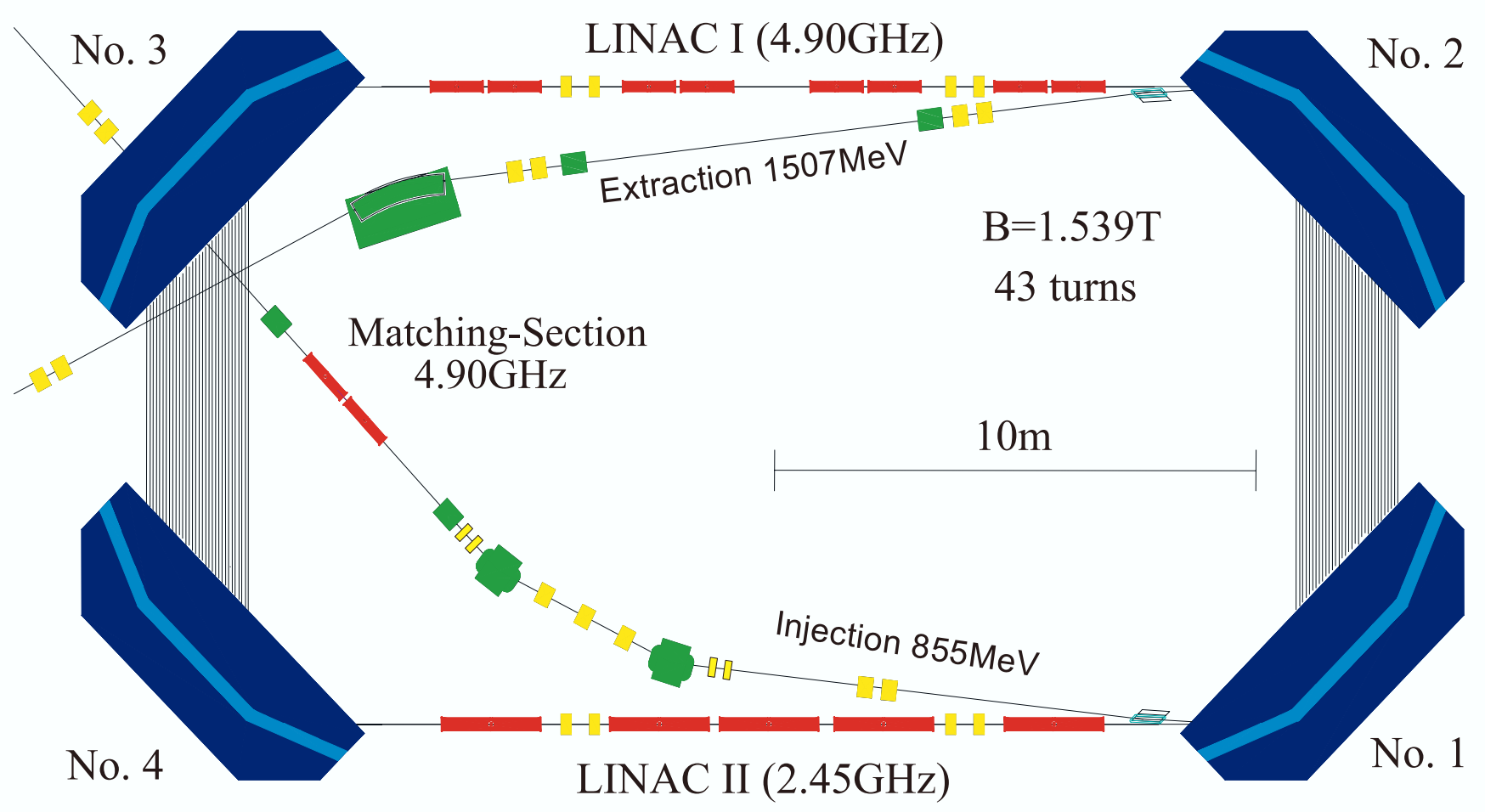

Fig. 6. Detailed scheme of the Harmonic Double Sided Microtron (HDSM) for MAMI C.

anomaly of the electron. Currents of $I>30 \mu \mathrm{A}$ with polarisations of $P>85 \%$ are now possible.

Apart from steady improvements of the degree of polarisation and the lifetime of the photocathodes, the installation of the harmonic $2 \mathrm{f}$-prebuncher at the injector linac and the rf-synchronised laser for up to $90 \%$ transmission efficiency made it possible to increase the MAMI operation with polarised electrons from $20 \%$ to now $60 \%$ of the total beam time satisfying the demands of the experiments.

\section{The Harmonic Double-Sided Microtron (HDSM) as the fourth stage of MAMI}

In 1999 a new Collaborative Research Centre (SFB443, "Many-body structure of strongly interacting systems") was founded, which for its "second stage" physics program demanded an electron beam of $1.5 \mathrm{GeV}$. The ideas for upgrading the MAMI energy had to consider as boundary conditions that the excellent beam quality and reliability of MAMIB must be preserved, that the new fourth stage had to fit into the existing buildings and that the research with the existing MAMIB had to go on without any longer shutdown periods. Moreover, considering the limited manpower capacity of the institute and the tight time schedule envisaged, it was evident that one had to base the new accelerator stage on the expertise of the institute. So very early the decision was taken to stay with the well proven and tested technology applied for the RTMs: normal conducting rf-accelerator structures and iron core magnets with normal conducting excitation coils.

The latter point clearly implied that one could not realise MAMI C as a fourth RTM. With iron core magnets one cannot increase the field strength very much beyond the $1.3 \mathrm{~T}$ of RTM3. The size and weight of the two such $180^{\circ}$ end magnets would grow with the cube of the maximum energy, i.e. to formidable weight of $450 \mathrm{t} \times(1.5 / 0.855)^{3}=2430 \mathrm{t}$ each. However, the RTM with one linac is not the only possible microtron. Already since 1979 H. Herminghaus and K.H. Kaiser developed ideas and designs for "higher order" microtrons called "Polytrons", as multi-turn recirculators with strong phase focusing $([22,23,24])$.

At the "bicyclotron" or Double-Sided Microtron (DSM) (see the scheme in fig. 6) the pole face area is reduced by a factor of $(\pi-2) / \pi$ compared to an RTM. Therefore, a $1500 \mathrm{MeV}$ DSM has roughly the same magnets weight as an $850 \mathrm{MeV}$ RTM. As the next step one must consider, however, that the dynamic coherence condition changes and for a DSM is given by

$$
\Delta E / \text { turn }=n \cdot \frac{e c B}{2(\pi-2)} \cdot 2 \lambda_{r f}=n \cdot \frac{e c B}{\pi-2} \cdot \lambda_{r f},
$$

and naturally one will take $n=1$ for the lowest possible path lengthening of $2 \cdot \lambda_{r f} /$ turn. With the parameters of RTM3 $\left(B=1.28 \mathrm{~T}, \lambda_{r f}=0.1224 \mathrm{~m}\right)$ one would need 


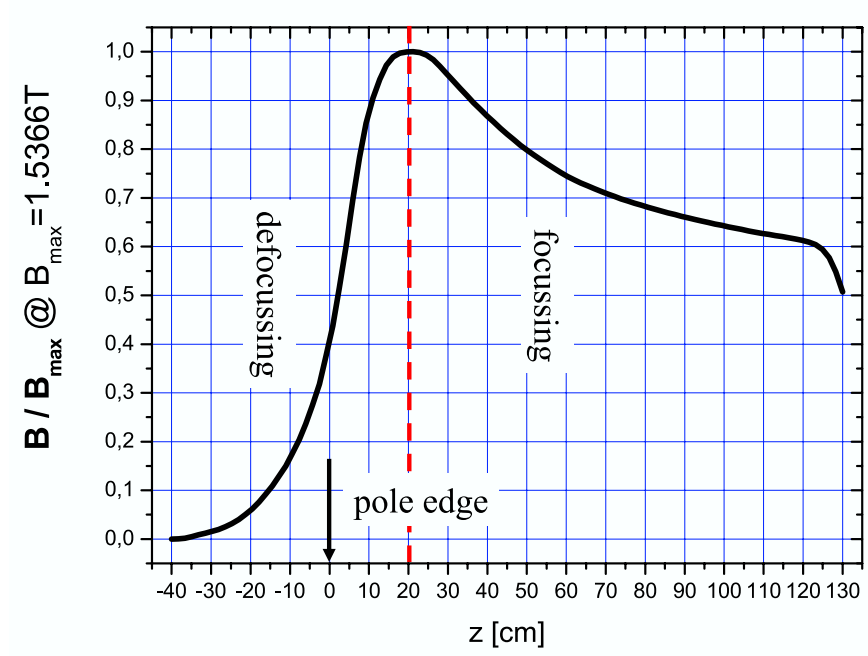

Fig. 7. Field gradient perpendicular to the pole edge of the $90^{\circ}$ dipoles of the HDSM for compensation of the vertical edgedefocusing.

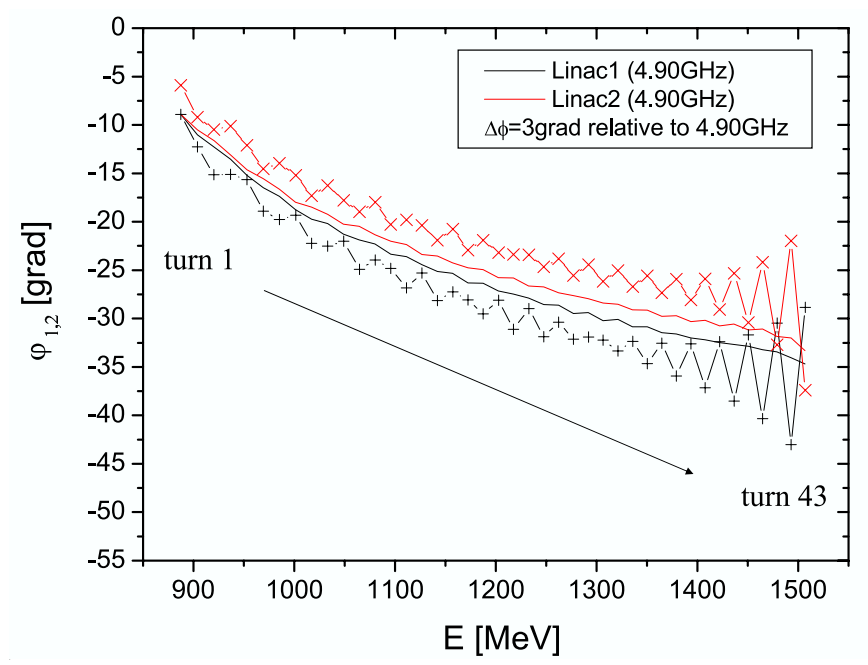

Fig. 8. Development of the synchronous phases of both linacs in DSM configuration for a phasing error between the two linacs of $3^{\circ}$.

$\Delta E=41.1 \mathrm{MeV} /$ turn. With the moderate well tested MAMI rf-gradient of $1 \mathrm{MV} / \mathrm{m}$ one would need $20 \mathrm{~m}$ long linacs, which would not fit into the existing buildings (fig. 12) and would moreover consume about four times the electric power of MAMIB. So it was evident that the frequency of the DSM had to be $4.90 \mathrm{GHz}\left(\lambda_{r f}=0.0612 \mathrm{~m}\right)$, with two about $10 \mathrm{~m}$ long linacs and with the other parameters similar to that of RTM3.

Concerning the transverse optics, the $45^{\circ}$ entrance and exit angles of the beam at the four $90^{\circ}$ magnets are with their strong vertical defocusing a very critical point. A detailed investigation [25] was done for several quadrupole configurations on the dispersive paths (quadrupole triplet / two quadrupole doublets on each half recirculation). It showed, that this way of compensation would work only in
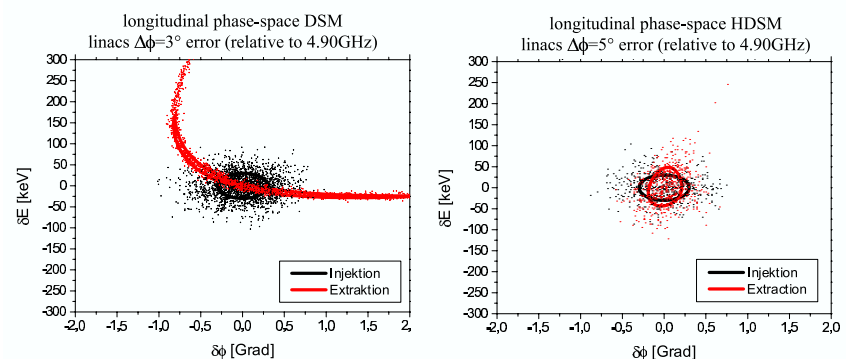

Fig. 9. Longitudinal input and output phase space for the DSM- / HDSM-configuration (left / right) with phasing errors of $3^{\circ} @ 4.90 \mathrm{GHz} / 5^{\circ} @ 4.90 \mathrm{GHz}$.

principle. To avoid strong distortions of the phase space, the quadrupoles must be extremely free of sextupole and other higher multipole errors and their individual setting and alignment for a dispersion free beam on the linac axis would be in practice a very cumbersome procedure. Therefore, it was decided to use the combined function solution, namely to introduce a magnetic field gradient perpendicular to the pole edge in the $90^{\circ}$ dipoles, which compensates for the edge-defocusing in the complete range of beam energies. The corresponding field profile is given in fig. 7 . Due to the field decay from $100 \%$ to $60 \%$, however, the mean field along the beam path decreases with increasing energy so that the necessary synchronous energy gain becomes lower and lower. As a consequence, the synchronous phase has to move away from the crest of the rf-waves in the linac in order to fulfil the dynamic coherence condition (eq. (7)). This happens automatically and smoothly by the longitudinal focusing if input energy and linac phases are optimised with respect to minimum phase oscillations. For an injection phase of $-8^{\circ}$ one will end at $-34^{\circ}$ for turn 43. This is well within the phase stable range of the DSM $\left(-4<n \cdot \pi \cdot \operatorname{tg}\left(\varphi_{s}\right)<0\right.$, i.e. $-51.9^{\circ}<\varphi_{s}<0^{\circ}$ for $\left.n=1\right)$, however, only for the ideally symmetric DSM, where one half turn can be considered as a machine period. If the symmetry is perturbed, e.g. by a wrong phase setting of one of the linacs, the DSM can be considered as spliting into two RTMs with a stable phase boundary of $-32.5^{\circ}$. From fig. 8, assuming a phase error of only $3^{\circ}$ between the two linacs, one sees that only the last ca. 10 turns are affected by this stopband. However, more detailed tracking calculations showed that the acceptance of the DSM is distinctly diminished (fig. 9, left). Therefore, a standard DSM with gradient dipole magnets would have to be operated with extreme care for its symmetry, e.g. a phase change of $1^{\circ}$ at $4.9 \mathrm{GHz}$ would mean a change of a steering cables effective length of only $0.1 \mathrm{~mm}$.

However, because the reason for this unstable region is finally the too strong longitudinal focusing when the electrons slip down on the $4.9 \mathrm{GHz}$ wave, a glance at the "time table" of the bunches in the two linacs of a DSM with sub-harmonic injection showed a way out of this difficulty (fig. 10). One can see, that in one of the linacs only every second bucket is populated by the recirculated bunches. So one could operate here with the MAMIB frequency 


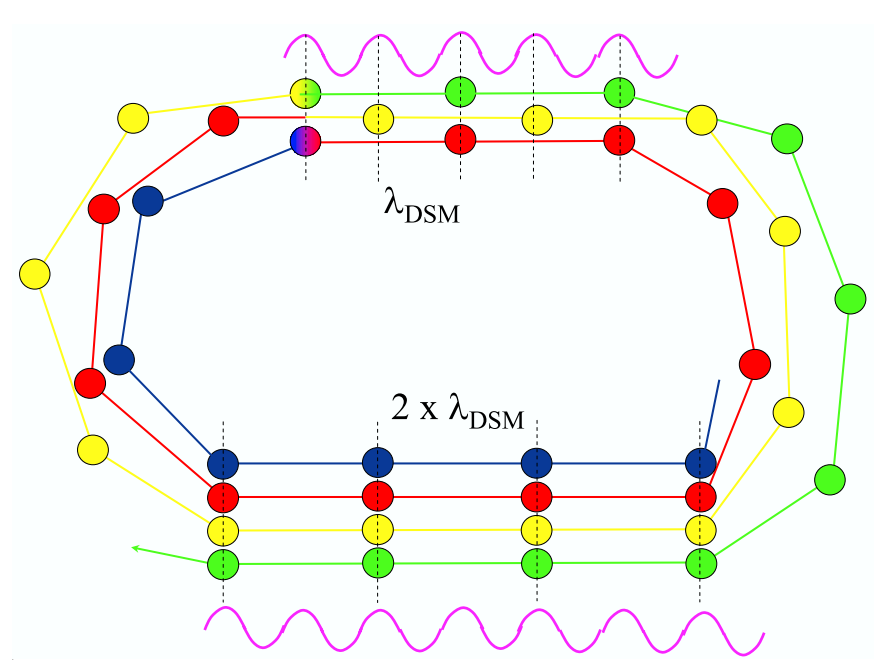

Fig. 10. Simplified scheme of the bunch arrival time in a DSM operating with a sub-harmonic injection frequency.

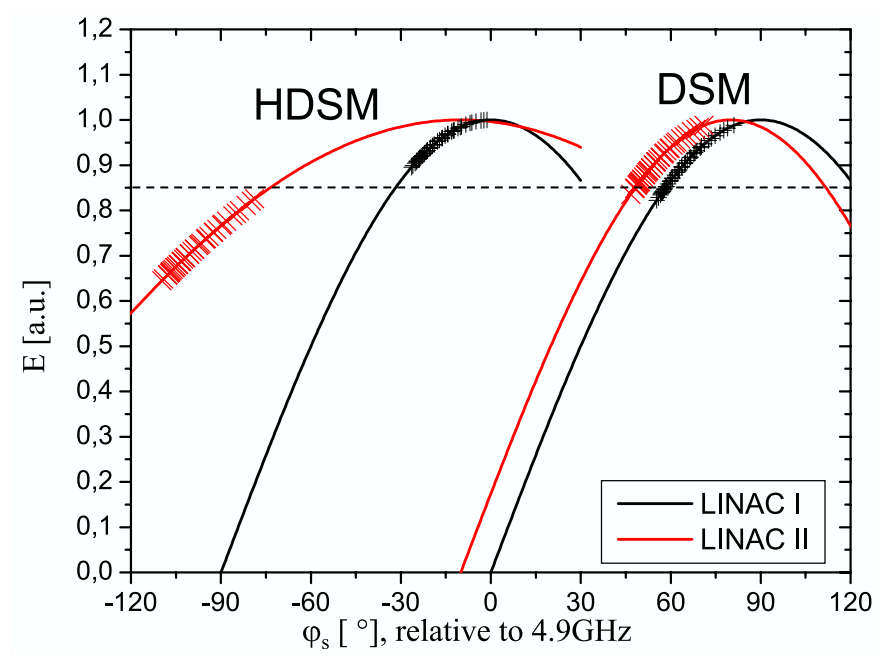

Fig. 11. Bunch phase migration in the DSM (phase scale shifted by $90^{\circ}$ ) and HDSM configuration. Clearly the instable area of the $4.9 \mathrm{GHz}$ wave can be avoided only in the HDSM scheme.

of $2.45 \mathrm{GHz}$, and it turned out, that by an appropriate amplitude and phase relation between the $2.45 \mathrm{GHz}$ and $4.9 \mathrm{GHz}$ wave most of the necessary reduction of the energy gain can be overtaken by the low frequency linac with its less steep gradient (fig. 11). On the $4.9 \mathrm{GHz}$ wave the bunches migrate now only from $0^{\circ}$ to ca. $-26^{\circ}$ and so stay distinctly away from the dangerous $-32.5^{\circ}$.

This Harmonic Double-Sided Microtron (HDSM, [26]) shows a good longitudinal stability, e.g. a phase error of more than $5^{\circ}$ between the two linacs is tolerable (fig. 9, right). Concerning the transverse optics, due to the moderate total energy gain factor of the HDSM of only 1.75 , it is possible to stay with the horizontal and vertical beta-functions below $20 \mathrm{~m}$ during the acceleration process with just two quadrupole doublets on each linac axis. Tracking calculations taking into account the ef- fect of emittance growth due to quantum fluctuations of synchrotron radiation ${ }^{1}$ [27], show that the normalised longitudinal/horizontal emittances increase only by a factor of $2 / 1.5$. Therefore, the absolute emittance will stay nearly constant and the beam sizes on the linac axes are in the order of only some tenths of a millimetre. The final design parameters for the HDSM are given in table 1, its scheme in fig. 6 and its floor plan in fig. 12.

The MAMIB frequency of $2.45 \mathrm{GHz}$ was in a wellestablished industrial heating band, with many components available from the shelve, whereas at $4.9 \mathrm{GHz}$ there existed nearly no high power rf-components. Therefore, here many developments had to be started. To have a quick start of production for the five rf-sections needed for the $2.45 \mathrm{GHz}$ linac, it was decided to make them as copies of the well tried ones at RTM3 [28] (with a slightly adapted length, 33 accelerating cells (AC) instead of 29). The contract was given to the INP of the Moscow State University collaborating with the klystron firm TORIY, because of their great experience in developing and optimising the biperiodic on-axis coupled structures. However, because of many technical failures (vacuum leaks, contaminations in the sections resulting in severe multipacting effects) only three sections were finally delivered. The contract had to be terminated, and the remaining sections were produced by the company ACCEL Instruments (Bergisch Gladbach, Germany) [29] without greater difficulties, apart from also some multipactor problems.

Concerning the $4.9 \mathrm{GHz}$ accelerating structures more time was available because of the lack of a high-power klystron at the beginning. So an intensive optimising work was done at IKPh. For the quite tiny cavities the relative mechanical tolerances had to be relaxed for a promising industrial series production. This affected mainly the cellto-cell coupling increasing it by a factor of two $\left(k_{2.45 \mathrm{GHz}}=\right.$ $4 \%, k_{4.9 \mathrm{GHz}}=8.8 \%$, [30]). As for their modified geometry there remained uncertainties, e.g. concerning multipactoring, and a prototype was successfully built and full high power tests were performed at the IKPh [31]. The series production of these very well behaving structures was done without any difficult problems again in cooperation with ACCEL.

Concerning the high power rf-sources for MAMI C, the $2.45 \mathrm{GHz}$ klystron posed no fundamental problem. The tube (TH2174) was delivered by THALES Electron Devices as an improved and modernised version with better electron beam focusing of the old TH2075 used at MAMI B.

At $4.9 \mathrm{GHz}$ a new klystron had to be developed, and offers were asked from THALES and CPI/Varian. No problems were expected, because the power-limiting curves given in the literature [32] asserted, that at this frequency cw-klystrons up to several $100 \mathrm{~kW}$ could be built. Quite ample specifications were given to the factories $(60 \mathrm{~kW}$ $\mathrm{cw}$ for feeding two $4.9 \mathrm{GHz}$ sections, $\geq 55 \%$ efficiency and $\geq 47 \mathrm{~dB}$ gain), also with respect to a later energy upgrade

\footnotetext{
1 These effects scale with $E^{5-7}$ and, therefore, with a beam energy in the order of $1 \mathrm{GeV}$ their influence on the beam acceleration needs to be carefully investigated.
} 


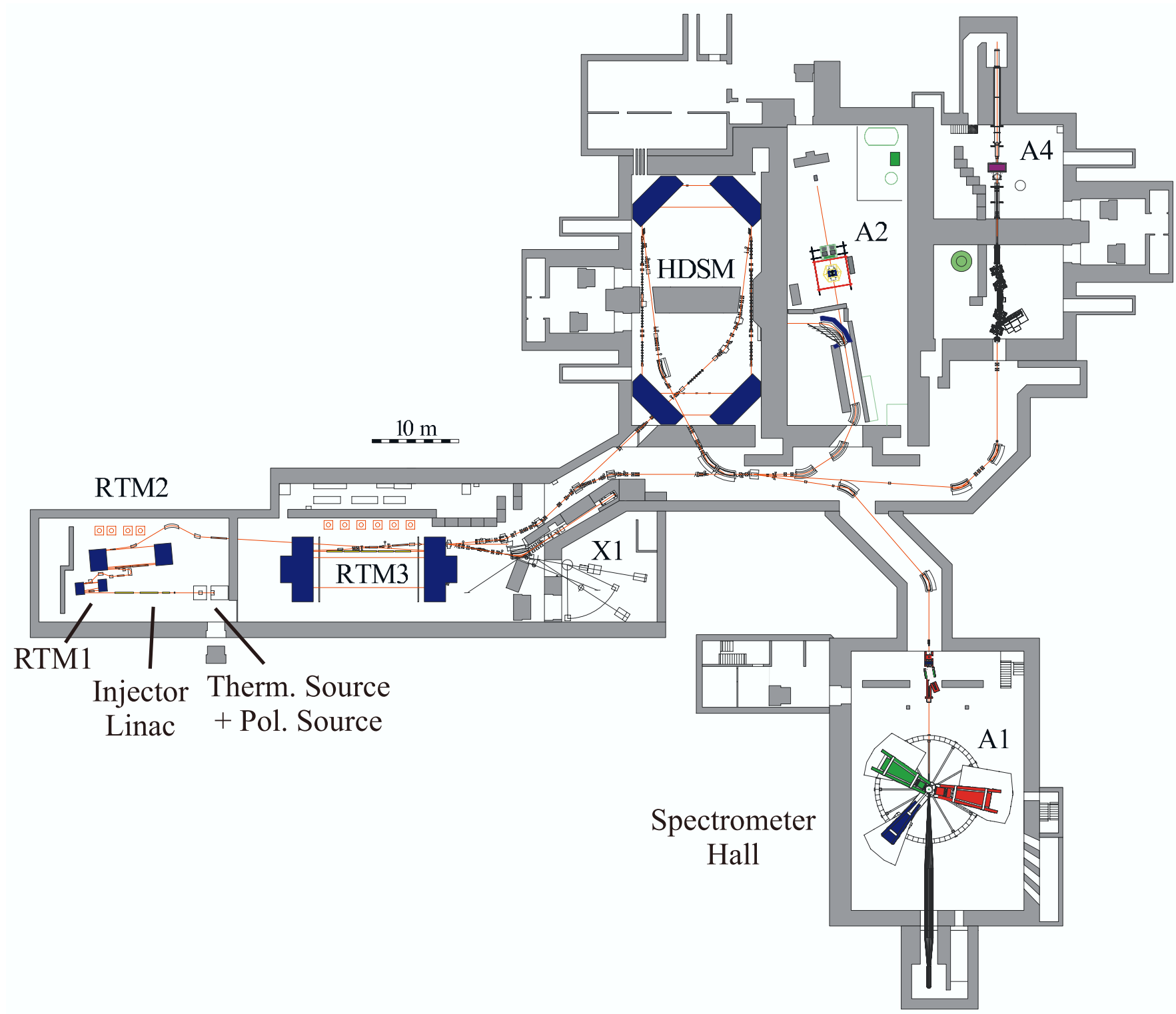

Fig. 12. Floor plan of MAMIC. The installation up to RTM3 (MAMIB) has not been changed essentially since 1990 (A2: Tagger / A4: Parity Violation / X1: Radiation Physics, till 2000 in the HDSM Hall).

of the HDSM. The contract was given to THALES. Considering both bidders would have caused an increase of the total costs of this system of more than $50 \%$. But obviously the problems for fabricating a power klystron at this high frequency were underestimated. It took a long series of prototypes - partly damaged by trivial technical failures - and 27 months compared to the anticipated 12 months delivery time, before the desired tube was in house and could be used for the power tests of many other $4.9 \mathrm{GHz}$ components (rf-structures, circulators, special waveguide components). These tests were successfully performed in 2003. However, the prototype TH2166-tube showed strong multipacting discontinuities on its transfer curve and was therefore not qualified for precision operation at the accelerator. THALES could solve the multipacting problem at the next prototype by Ti-coating the nose cones of the klystron resonators, but unfortunately through the higher surface resistivity thermal problems occurred. Finally, with a total delay of 26 months all tubes needed for MAMI C were delivered, fortunately, with a production guarantee for the TH2166 tube till 2010 by THALES. However, with somewhat reduced specifications $(50 \mathrm{~kW}$, $\geq 45 \%$ efficiency and multipactor freeness only for somewhat restricted operating conditions), which is just safely adequate for the $1.5 \mathrm{GeV}$ operation of the HDSM.

All other high power components, e.g. the circulators by AFT and the water loads from Spinner, worked satisfactorily from the beginning. The two $30 \mathrm{kV} / 27 \mathrm{~A}$ klystron power supplies built by BRUKER, Wissenbourg, were successfully operated in several longterm tests, 

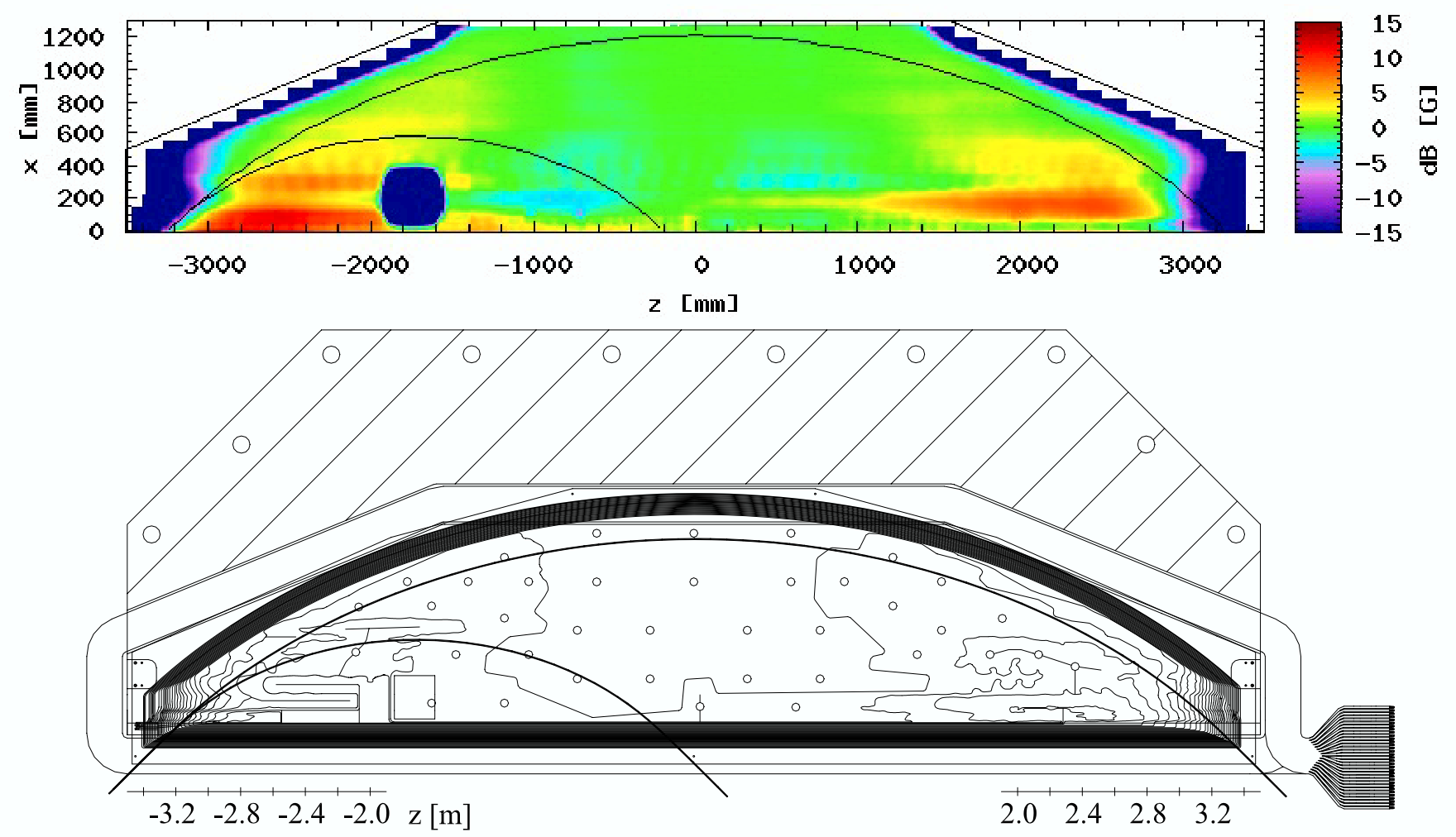

Fig. 13. Measured vertical $B$-field of HDSM dipole No.2 normalised to the ideal field gradient (top), and the construction drawing of the corresponding correction coil (bottom). The dark quadratic area at the lower left of the field map is due to a piece of parallel pole faces necessary for an NMR-probe for precise field regulation.

Table 2. Main parameters of the HDSM dipoles.

\begin{tabular}{|l|l|}
\hline field strength $[\mathrm{T}]$ & $1.53-0.95$ \\
\hline gap distance [mm] & $85-139$ \\
\hline mech. length of front edge $[\mathrm{m}]$ & 7 \\
\hline usable length of front edge $[\mathrm{m}]$ & 6.5 \\
\hline iron weight [t] & 250 \\
\hline coils copper profile outside $\left[\mathrm{mm}^{2}\right]$ & $12 \times 12$ \\
\hline coils cooling duct diameter $[\mathrm{mm}]$ & 8 \\
\hline number of windings & $2 \times 256$ \\
\hline current/voltage $[\mathrm{A} / \mathrm{V}]$ & $212 / 340$ \\
\hline copper weight $[\mathrm{t}]$ & 6.85 \\
\hline
\end{tabular}

especially during the high power conditioning and testing of the fully installed and commissioned $2.45 \mathrm{GHz}$ linac of the HDSM.

Naturally, beside the task to design and build up the worldwide first $4.9 \mathrm{GHz} \mathrm{cw}$ linac, the manufacturing of the four $90^{\circ}$-bending magnets with field gradient presented the second highly critical challenge for the completion of the HDSM. The mechanical and magnetic design of these dipoles was completely done at IKPh. The main goal was to get magnets with excellent field quality at minimum size (existing halls) and iron consumption $([33,34])$. The main parameters of these magnets are given in table 2 . The call for tender started in 1999 and in 2000 the contract was awarded to the French company USINOR ${ }^{2}$. Aside the promising manufacturing capabilities of this company the main argument was, that only USINOR offered to produce the magnets essentially of only two symmetric pieces (upper and lower piece), which is clearly the favourable geometry to avoid any discontinuities perpendicular to the pole edge. The magnet pieces, each weighing $125 \mathrm{t}$, were casted out of high permeable iron and then machined at the company SFAR, a subcontractor of USINOR. This machining procedure for a high quality and precise surface of the partly concave pole pieces was worked out in close collaboration with IKPh. Due to the complicated pole geometry it was expected, that for the final field correction of the magnets to the $10^{-4}$ level not only symmetric, but also asymmetric field errors (resulting in unwanted field components in the plane of beam acceleration) must to be corrected. Based on the well proven concept of surface correction currents [35], a procedure had been developed which allows to extract the symmetric and asymmetric field components by a simultaneous measurement of the vertical magnetic field in and $\pm 25 \mathrm{~mm}$ out of the midplane of the magnets, and to construct surface correction coils which compensate both errors simultaneously $([36,37])$. The first magnet was delivered end of 2001 and all four magnets were finally in place end of 2002. The contract for

2 Today SFAR STEEL (Le Creusot, France). 
the manufacturing of the excitation coils was awarded to the company SIGMAPHI (Vannes, France). They introduced a special bi-filar winding technology, which allowed to realise optimum heat distribution within the coils, to avoid internal brazing and to choose reasonable power supply parameters [34]. Both guarantees a high reliability over the lifetime of the accelerator. Each magnet is fed by an individual, highly stabilised power supply $(478 \mathrm{~V}, 260 \mathrm{~A}$, short term/long term stability: $3 \mathrm{ppm} / 10 \mathrm{ppm}$ ) manufactured by DANFYSIK (Jyllinge, Denmark). By feeding back the reading of NMR-probes to the PS, the field of each magnet is stabilised to better than $10^{-5}$.

It took till September 2003 to finish all magnet field measurements. To explore the capabilities of the magnets for a later energy upgrade, these measurements were not only done at the nominal field of $1.53 \mathrm{~T}$ but also at $1.64 \mathrm{~T} \cong 1.61 \mathrm{GeV}$ and even $1.71 \mathrm{~T} \cong 1.67 \mathrm{GeV}$. In fig. 13 the measured field of dipole No.2 (at the nominal field of $1.53 \mathrm{~T}$ normalised to the ideal field gradient) is plotted. In the central area of the magnet the field deviations are already in the order of $10^{-4}$, a clear proof of the excellent work done by USINOR/SFAR. As a further result of this high manufacturing precision, the analysis of the asymmetric field errors of the magnets showed, that the transverse components are well below $1 \mathrm{mT}$. A rough estimation of the influence of the resulting vertical beam deflections of $0.1 \mathrm{mrad}$ to max. $0.35 \mathrm{mrad}$ leads to an acceptable coupling of only a few percent between the horizontal and vertical phase spaces. So it was decided to do the final correction only for symmetric field errors, resulting in much simpler identical upper and lower correction coils. In the lower part of fig. 13 a sketch of one of this correction coils, manufactured by water jet cutting of a $3 \mathrm{~mm}$ thick aluminium plate, is shown. With these pairs of coils the desired field accuracy of $2 \times 10^{-4}$ was easily achieved for all four magnets. One can clearly see, that most of the correction must be done near the corners of the magnet, because here quite large field decays exist. This behaviour was already predicted by TOSCA-simulations and are due to the triangular cut necessary to fit the magnets as far as possible into the corners of the accelerator hall. It turned out, that even at the design field level of $1.53 \mathrm{~T}$ this field decay leads to deflection errors of up to $2.2 \mathrm{mrad}$ at low electron energies. Because it reaches far into the fringe field region, it cannot be corrected by surface correction coils alone. Therefore, at the entrance and exit corner of each dipole individually designed vertical iron shims attached to its front face are necessary. Together with the steering magnets on the return paths and the linac axis they will provide a proper angle and position correction of the beam [37]. Because with increasing field of the dipoles the field decay at the magnet corners gets much stronger, a later energy upgrade of the HDSM, based on the experiences gained during the operation at $1.5 \mathrm{GeV}$, will most probably require the construction and installation of a fully new set of correction coils and iron shims.

Presently, all four dipole magnets are aligned and equipped with their individual set of correction coils and vacuum chambers. The $2.45 \mathrm{GHz}$ linac is commissioned and ready for operation, whereas the installation of the $4.90 \mathrm{GHz}$ linac, after the final delivery of the 10 needed accelerator sections, has just started. The next step is the installation of the two recirculation path vacuum systems and the completion of the injection and extraction beam lines. The first operation of the HDSM is expected in the first half of 2006. After a period of commissioning in diagnostic pulse mode with low beam power $(10 \mathrm{~ns}$, high-intensity bunch trains with a repetition rate of max. $10 \mathrm{kHz}$ ), very soon the first physics experiments will be started since all upgrades of the beams lines, the photon tagger and the spectrometers has been finished.

Many people worked together to realise the very successful operation of MAMI over the last 25 years. Here I just want to mention the certainly most important ones: Helmut Herminghaus, who is the intellectual father of MAMI and laid the strong foundations for this success story and KarlHeinz Kaiser, who overtook the responsibility for the MAMI operation and development in the early 1990s and set the guidelines for the future of MAMI: the design and installation of the Harmonic Double-Sided Microtron. The construction and operation of MAMI would not have been possible without Hans Euteneuer who is, amongst others, responsible for the developments in the rf-field. His great help for the preparation of this manuscript must be stressed as my last point.

\section{References}

1. J. Haimson, Linear Accelerators, edited by P.M. Lapostolle, A.L. Septier (Amsterdam, 1970) p. 415.

2. D. Husmann, IEEE Trans. Nucl. Sci. NS-30, No. 4, 3252 (1983).

3. L. Harwood, Proceedings of PAC2003, Portland, OR, USA (2003) p. 586.

4. E.M. Moroz, Sov. Phys. Dokl. 1, 326 (1956).

5. A. Roberts, Ann. Phys. (N.Y.) 4, 115 (1958).

6. B.H. Wiik et al., Linear Accelerators, edited by P.M. Lapostolle, A.L. Septier (Amsterdam, 1970) p. 553.

7. H. Herminghaus et al., Nucl. Instrum. Methods 138, 1 (1976).

8. H. Herminghaus et al., Nucl. Instrum. Methods 187, 103 (1981).

9. P. Axel et al., IEEE Trans. Nucl. Sci. NS-24, No. 3, 1133 (1977).

10. O. Hanson, Charlottesville Conference Paper Q (1979).

11. H. Herminghaus et al., Nucl. Instrum. Methods 163, 299 (1979).

12. H. Euteneuer et al., Proceedings of LINAC84, Seeheim, Germany (1984) p. 394.

13. M. Begemann et al., Nucl. Instrum. Methods 201, 287 (1982).

14. U. Schmidt-Rohr, Die Deutschen Teilchenbeschleuniger (U. Schmidt-Rohr, Heidelberg, 2001) p. 144.

15. H. Euteneuer et al., Proceedings of EPAC88, Rome, Italy (1988) p. 550.

16. H. Euteneuer et al., Proceedings of LINAC92, Ottawa, Canada (1992) p. 356.

17. H.J. Kreidel, $\mathrm{PhD}$ Thesis, KPH 12/87, University of Mainz, Mainz, Germany (1987). 
18. M. Seidl, Proceedings of EPAC2000, Vienna, Austria (2000) p. 1930.

19. K. Aulenbacher et al., Journal AIP, Vol. 675 (2002) 1088.

20. H. Steffens PhD Thesis, KPH 01/94, University of Mainz, Mainz, Germany (1994).

21. V.I. Shvedunov et al., Proceedings of EPAC96, Barcelona, Spain (1996) p. 1556.

22. K.H. Kaiser, Proceedings of the Conference on Future Possibilities for Electron Accelerators, Charlottesville, VA, USA (1979) V-1.

23. H. Herminghaus et al., Proceedings of LINAC81, Santa Fe, N.M., USA (1981) p. 260.

24. H. Herminghaus, Nucl. Instrum. Methods A 305, 1 (1991).

25. S. Ratschow, PhD Thesis, KPH 02/00, University of Mainz, Mainz, Germany (2000).

26. A. Jankowiak et al., Proceedings of EPAC02, Paris, France (2002) p. 1085.

27. J. Herrmann et al., Proceedings of PAC99, New York, USA (1999) p. 2915.
28. H. Euteneuer et al., Proceedings of LINAC86, Stanford, CA, USA (1986) p. 508.

29. H. Euteneuer et al., to be published in Proceedings of EPAC06, Edinburgh, GB (2006).

30. H. Euteneuer et al., Proceedings of EPAC00, Vienna, Austria (2000) p. 1954.

31. A. Jankowiak et al., Proceedings of LINAC04, Lübeck, Germany (2004) p. 842.

32. G. Faillon et al., Proceedings of LINAC86, Stanford, CA, USA (1986) p. 122.

33. U. Ludwig-Mertin et al., Proceedings of EPAC98, Stockholm, Sweden (1998) p. 1931.

34. A. Thomas et al., Proceedings of EPAC02, Paris, France (2002) p. 2379.

35. H. Herminghaus, Proceedings of EPAC88, Rome, Italy (1988) p. 1151.

36. M. Seidl et al., Phys. Rev. STAB 5, 062402 (2002).

37. F. Hagenbuck et al., Proceedings of EPAC04, Lucerne, Switzerland (2004) p. 1669. 